\title{
NUEVOS REGISTROS DE DEPREDACIÓN POR BOA CONSTRICTOR(SERPENTES: BOIDAE) EN COLOMBIA
}

NEW RECORDS OF PREDATION BY BOA CONSTRICTOR (SERPENTES: BOIDAE) IN COLOMBIA

\author{
Juan Manuel Acosta-Ortiz ${ }^{1,2^{*}}$, Juan Sebastián Bobadilla-Molina ${ }^{1,2}$, Jesús Alejandro GonZÁles- \\ ROJAS $^{1,2}$ \& SANTIAGO MARTÍNEZ-VÉLEZ ${ }^{1}$ \\ ${ }^{1}$ Departamento de Biología y Química. Programa de Biología. Universidad de los Llanos. Villavicencio. Meta. Colombia. \\ ${ }^{2}$ Semillero de Investigación BioHerp. Universidad de los Llanos. Villavicencio. Meta. Colombia. \\ Correspondence: juan.acostaherp@gmail.com \\ Received: 2020-11-29. Accepted: 2021-03-26.
}

Abstract.- Two cases of predation by Boa constrictor in Meta department (Colombia) were reported. The first record is of Psarocolius decumanus as new food item, while the second is a predation record on Ameiva sp.

Keywords.- Diet, red-tailed boa, crested oropendola, whiptail lizard.

Resumen.- Se reportan dos casos de depredación por Boa constrictor en el departamento del Meta (Colombia). El primer registro de Psarocolius decumanus como nuevo item alimenticio y un evento de depredación de Ameiva sp.

Palabras clave.- Dieta, boa de cola roja, oropéndola crestada, lagartija cola de látigo.

Boa constrictor Linnaeus, 1758 es una serpiente que se encuentra ampliamente distribuida en Sudamérica, además de presentar poblaciones introducidas en Centroamérica y el sureste de Estados Unidos (Snow et al., 2007; Reynolds \& Henderson, 2018; Uetz et al., 2020). Su dieta ha sido documentada en poblaciones nativas e introducidas, donde se han reportado presas endotérmicas y ectotérmicas, como reptiles, aves y mamíferos. (Beebe, 1946; Martins \& Oliveira, 1998; Sironi et al., 2000; Quick et al., 2005; Pizzato et al., 2009).

En Colombia dos especies del género Boa han sido reportadas, Boa imperator cuya distribución comprende la zona occidente y Boa constrictor, que es registrada en el resto del país (Card et al., 2016). En el oriente del país, B. constrictor ha sido registrada en localidades donde predominan formaciones vegetales como bosques, morichales, matorrales y pastizales, lo que puede generar una oferta alimenticia amplia para la especie (RangelCh \& Minorta-Cely, 2014; Trujillo-P et al., 2014). En esta nota se reportan dos eventos de depredación por B. constrictor para el oriente del país, en el departamento del Meta, en los municipios de Mapiripán y Villavicencio.

El 29 de septiembre del 2020 a las 9:20 horas, en la finca Zaragoza $\left(2.886944^{\circ} \mathrm{N}, 72.167222^{\circ} \mathrm{W}\right.$; elev. $181 \mathrm{~m}$ s.n.m.) ubicada en el municipio de Mapiripán, fue observado una Boa constrictor ingiriendo un individuo de Psarocolius decumanus (Icteridae)
(Fig. 1). Se observó que la serpiente constreñía el cuerpo del ave, mientras la tragaba desde la cabeza. Al detectar la presencia de los observadores, la serpiente empezó a regurgitar la presa, por lo que la observación directa fue interrumpida. A pesar de esto, la presa no fue regurgitada completamente. Pasados aproximadamente 30 minutos, la serpiente ya había ingerido el ave en su totalidad. Este evento de depredación se convierte en el primer caso documentado de esta especie de ave en la dieta de B. constrictor.

El segundo reporte se registró en el campus de la Universidad de los Llanos ( $4.074417^{\circ} \mathrm{N}, 73.584944^{\circ} \mathrm{W}$; elev. $398 \mathrm{~m} \mathrm{s.n.m.)}$, en el municipio de Villavicencio. Este campus presenta formaciones vegetales boscosas, áreas de cultivos, estanques piscícolas, canales de agua artificiales y edificaciones. En la zona de edificaciones, un individuo de Ameiva sp. (Teiidae) fue cazado por un individuo de Boa constrictor de aproximadamente $60 \mathrm{~cm}$, a las 10:00 horas del 27 de junio del 2019 (Fig. 2). La boa se encontraba bajo una llanta de vehículo, que era usada como maceta para una planta ornamental, cuando el individuo de Ameiva sp. se desplazó frente a la serpiente, esta lo capturo con una mordida en la zona del cuello y seguidamente lo atrapó con su cuerpo, realizando constricción por aproximadamente 20 minutos. Posteriormente, la serpiente ubicó la cabeza del lagarto y lo comenzó a ingerir. Durante la ingesta, la serpiente presentaba leves dificultades al engullir las extremidades del lagarto. Una 


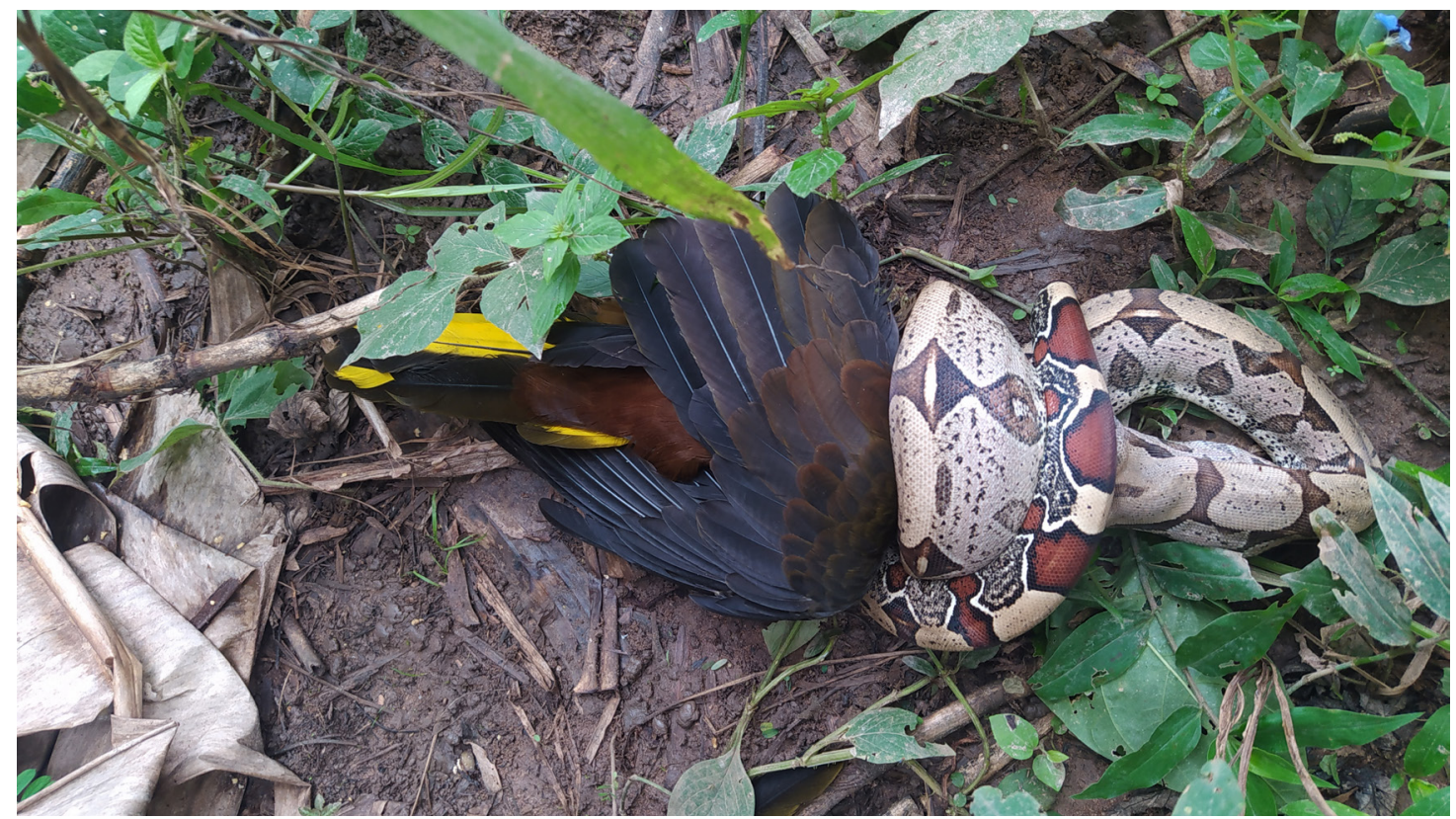

Figure 1. Predation of Psarocolius decumanus by Boa constrictor in Mapiripán (Meta), Colombia. Photo: Santiago Martínez.

Figura 1. Depredación de Psarocolius decumanus por Boa constrictor en Mapiripán (Meta), Colombia. Foto: Santiago Martínez.

vez ingerida completamente la presa, la boa se refugió bajo la llanta donde en un principio se encontraba, pero al ser una zona de transcurrir constante de personas, fue reubicada en el área boscosa del campus, donde la serpiente se refugió bajo un tronco. Durante el traslado de un sitio al otro, la serpiente no presentó señales de estrés, ni regurgitó la presa.

Se ha registrado que las boas buscan sitios que frecuentan sus presas para poderlas emboscar eficazmente (Montgomery \& Rand, 1978; Sironi et al., 2000; Rocha-Santos et al., 2014). Por lo que, la observación de la serpiente bajo la maceta, sugiere que el depredador ya venía acechando el lagarto, o podría estar apareciendo que apareciera cualquier presa. Adicionalmente, el género Ameiva ya ha sido mencionado en la dieta de $\mathrm{B}$. constrictor en Brasil (Cunha \& Nascimento, 1978; Pizzato et al., 2009; Bernarde y Abe, 2010; Silva et al., 2016), Guyana y Venezuela (Beede, 1946) y en una población introducida en la isla de Aruba (Quick et al., 2005), donde parece ser que los lagartos son un componente importante en la dieta de la especie.

\section{CITED LITERATURE}

Beebe, W. 1946. Field notes on the snakes of Kartabo, British Guiana, and Caripito, Venezuela. Zoologica 31:11-2.
Bernarde, P.S. \& A.S. Abe. 2010. Hábitos alimentares de serpentes em Espigão do Oeste, Rondônia, Brasil. Biota Neotropica 10:167173.

Card, D.C., D.R. Schield, R.H. Adams, A.B. Corbin, B.W. Perry, A. L. Andrew, G.J.M. Pasquesi, E.N. Smith, T. Jezkova, S.M. Boback, W. Booth \& T.A. Castoe. 2016. Phylogeographic and population genetic analyses reveal multiple species of Boa and independent origins of insular dwarfism. Molecular Phylogenetics and Evolution 102:104-116.

Cunha, O. R. \& F.P. Nascimento. 1978. As cobras da região leste do Pará. Publicações Avulsas Museu Paraense Emilio Goeldi 31:1218.

Martins, M. \& M.E. Oliveira. 1998. Natural history of snakes in forests of the Manaus Region, Central Amazonia, Brazil. Herpetological Natural History 6:78-150.

Montgomery, G.G. \&A.S. Rand. 1978. Movements, body temperature and hunting strategy of a Boa constrictor. Copeia 3:532-533.

Pizzatto, L., O.A.V. Marques \& K. Facure. 2009. Food habits of Brazilian boid snakes: overview and new data, with special reference to Corallus hortulanus. Amphibia-Reptilia 30:533-544. 


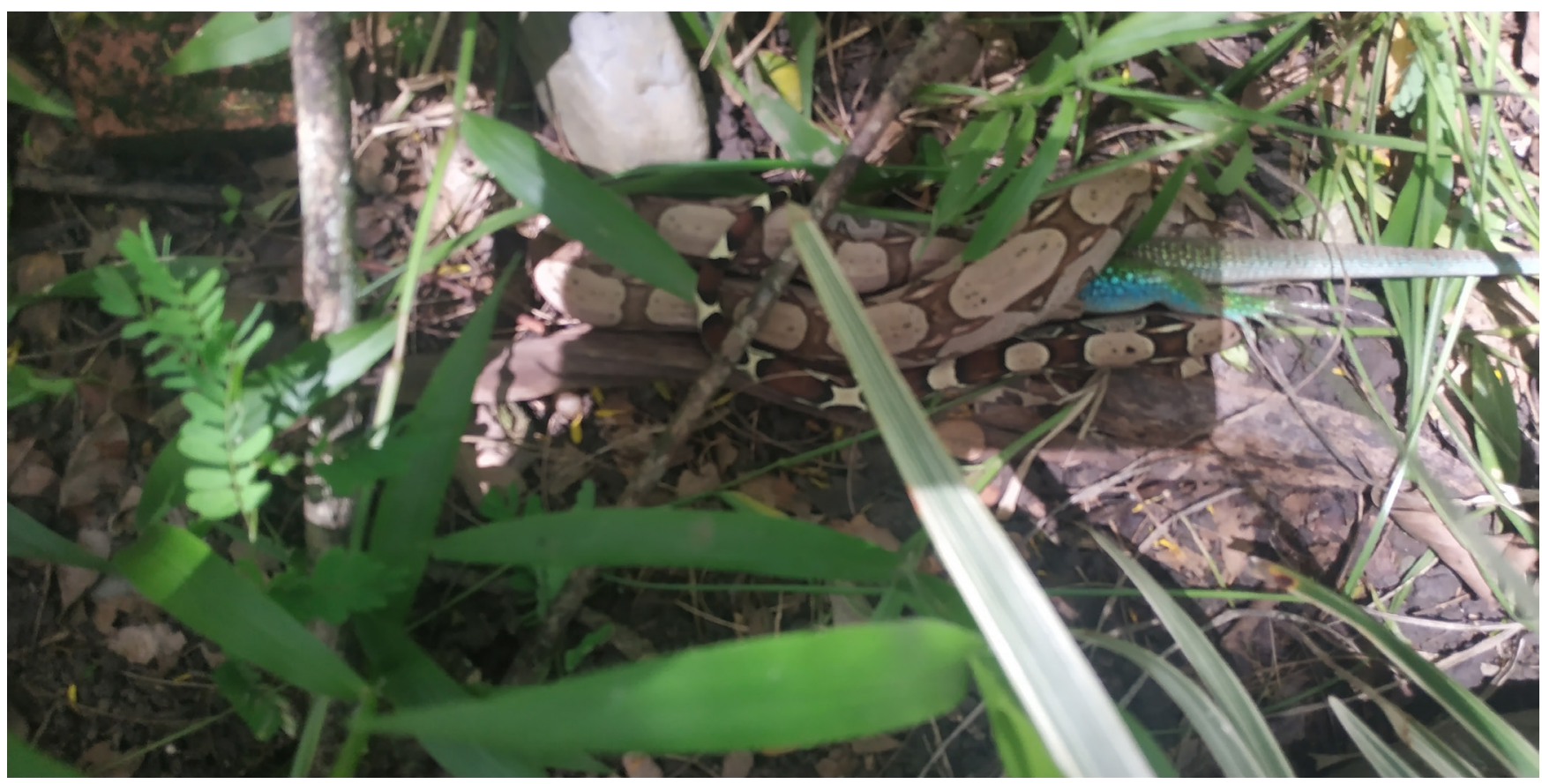

Figure 2. Predation of Ameiva sp. by Boa constrictor in Villavicencio (Meta), Colombia. Photo: Jesús Alejandro González.

Figura 2. Depredación de Ameiva sp. por Boa constrictor en Villavicencio (Meta), Colombia. Foto: Jesús Alejandro González.

Quick, J. S., H.K. Reinert, E.R. de Cuba \& R.A. Odum. 2005. Recent occurrence and dietary habits of Boa constrictor on Aruba, Dutch West Indies. Journal of Herpetology 39: 304-307.

Rangel-Ch, J.O. \& V. Minorta-Cely. 2014. Los tipos de vegetación de la Orinoquia Colombiana. Pp. 533-612. En: J.O. Rangel-Ch. (Ed.). Colombia Diversidad Biótica XIV. La región de la Orinoquia de Colombia. Universidad Nacional de Colombia-Instituto de Ciencias Naturales, Bogotá, Colombia.

Reynolds, R. G. \& R.W. Henderson. 2018. Boas of the World (Superfamily Booidae): A Checklist With Systematic, Taxonomic, and Conservation Assessments. Bulletin of the Museum of Comparative Zoology 162:1-58.

Rocha-Santos, G., E. Barbier, \& M.O. Bordignon. 2014. Sweet trap: Boa constrictor (Serpentes: Boidae) preying on passerines on Cecropia pachystachya (Urticales: Cecropiaceae) in fruiting period. Biota Neotropica 14:e20140003.

Silva, C. F., E.P. Alcantara, C.S.L. Matias, C.R. Oliveira \& R.W. Ávila. 2016. Ameiva ameiva (Giant Ameiva). Predation. Herpetological Review 47:292.
Sironi, M., M. Chiaraviglio, R. Cervantes, M. Bertona, \& M. Rio. (2000) Dietary habits of Boa constrictor occidentalis in the Cordoba Province, Argentina. Amphibia-Reptilia 21:226-232.

Snow, R. W., K.L. Krysko, K.M. Enge, L. Oberhofer, A. WarrenBradley \& L. Wilkins. 2007. Introduced populations of Boa constrictor (Boidae) and Python molurus bivittatus (Pythonidae) in southern Florida. P.p. 416-438. En: R.W. Henderson. \& R. Powell (Eds.). Biology of the boas and pythons. Eagle Mountain Publishing, Eagle Mountain, USA.

Trujillo-P, A., J.E. Carvajal-C \& J.O. Rangel-Ch. 2014. Reptiles de la Orinoquia Colombiana. P.p. 635-664. En: J.O. Rangel-Ch. (Ed.). Colombia Diversidad Biótica XIV. La región de la Orinoquia de Colombia. Universidad Nacional de Colombia-Instituto de Ciencias Naturales, Bogotá, Colombia.

Uetz P, Freed P, Hošek J. (2020). The Reptile Database. http://www. reptile-database.org, [Consultado en Noviembre 2020]

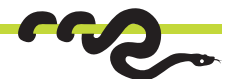

